\title{
Cesare Pavese: Dialoghi con il Cinematografo
}

Mariarosaria Fabris ${ }^{1}$

ABSTRACT: Quest'articolo presenta un aspetto meno noto dello scrittore Cesare Pavese: le sue riflessioni sul cinema in alcuni testi teorici e narrativi. PAROLE-CHIAVE: Cesare Pavese; cultura italiana; America.

ABSTRACT: Este artigo apresenta um aspecto menos conhecido do escritor Cesare Pavese: as suas reflexões sobre o cinema em alguns textos teóricos e narrativos.

PALAVRAS-CHAVE: Cesare Pavese; cultura italiana; America.

ABSTRACT: This article presents an unknown face of the writer Cesare Pavese: his reflections on cinema in some theoretical and narrative texts. KEYWORDS: Cesare Pavese; italian culture; America. 


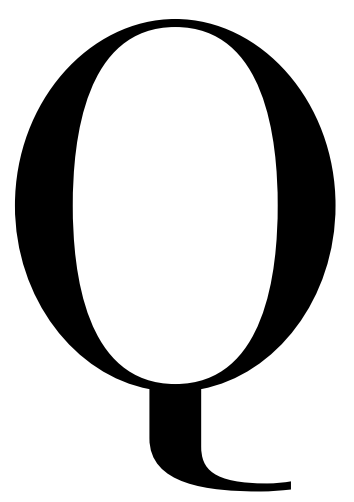

uando si pensa al dialogo fra Cesare Pavese e il cinema, vie ne subito in mente la sua sventurata relazione amorosa con Constance Dowling e la trasposizione cinematografica di Tra donne sole (1949) realizzata da Michelangelo Antonioni sotto il titolo de Le amiche (1955), con la collaborazione di Suso Cecchi d'Amico e Alba de Cespedes alla sceneggiatura. Meno conosciute la malia esercitata su di lui dalla settima arte, che Pavese ha espresso in racconti giovanili, la sua attività d'autore di soggetti cinematografici e le sue riflessioni teoriche.

Riprendendo un'attività interrotta dopo una prima esperienza nel 1928 (Un uomo da nulla), lo scrittore, nella primavera del 1950, si cimenta di nuovo nella stesura di soggetti cinematografici, fra cui Il diavolo sulle colline (8 marzo), Le due sorelle (o Breve libertà o Gioventù crudele, 18 marzo), Amore amaro (12 maggio) e Il serpente e la colomba (o La vita bella, 8-11 giugno), considerato "il più sviluppato e riuscito" (MASOERO, 2003, p. 22) $)^{2}$. Con il primo, l'autore intende rispondere alle critiche ne-

2. I quattro soggetti sono stati pubblicati da Cinema Nuovo tra il 1959 e il 1967. In Brasile sono reperibili solo i primi due. 
gative mosse al racconto omonimo e ribadire il suo parere favorevole su quell'opera. Gli altri tre li scrive per due giovani attrici americane, Doris Dowling e sua sorella, la "folgorante" Constance.

Il soggetto del 18 marzo 1950 narra la storia di Clara e Rosetta, due sorelle innamorate di Guido, un "individuo sradicato del dopoguerra" (PAVESE, 1959, p. 396), in bilico fra l'ambiente borghese e la malavita. Ambientato in un paesaggio ricorrente nella narrativa dello scrittore (una metropoli, una cittadina della Riviera e una città di provincia circondata dalla campagna), Le due sorelle risente di un tono eccessivamente melodrammatico riscontrabile in alcune opere cinematografiche neorealiste come $I l$ bandito (1946) di Alberto Lattuada o Riso amaro (1949) di Giuseppe De Santis.

In confronto al racconto lungo o romanzo breve omonimo scritto nel 1948, Il diavolo sulle colline del 1950 risulta un po' schematico, forse perché si tratta appunto di un soggetto e non di una sceneggiatura. In questo caso, la mancanza dei dialoghi, i quali, come ricorda Sergio Pautasso (1980, p. 94), hanno una "funzionalità essenziale" nell'opera pavesiana - "Per il dialogo, sono uno dei maestri riconosciuti del genere (!)" 3 -, e lo spezzettamento della trama in brevi capitoli ${ }^{4}$ ne impoveriscono la struttura narrativa. Anche i personaggi, in specie quello femminile, appaiono molto semplificati e l'aspetto mitico sembra essersi diluito.

Quasi sempre poco sviluppati o appena abbozzati, i soggetti sono spesso corredati da "osservazioni”" (così le chiama l'autore), le quali, come sottolinea la Masoero (2003, p. 25), "chiariscono l'intento dello scrittore e offrono la chiave di lettura del testo". Consapevole di non dominare la terminologia cinematografica, Pavese riconosce di avere una gran difficoltà a stendere una sceneggiatura vera e propria, per cui si limita a raccontare una storia "in una semplice prosa descrittiva, [...] come una novella", cioè a "scrivere semplicemente come uno scrittore, un narratore [...] e poi, se la storia andrà bene a quei tipi, discuteremo su chi sia più adatto a sviluppare una sceneggiatura"

Meno noti dei soggetti sono i testi giovanili che Pavese dedica al cinematografo, pubblicati da Massimo Mila alcuni anni dopo la scomparsa dell'amico: "I problemi critici del cinematografo", scritto fra il 28 maggio e il 6 giugno 1929, e "Di un nuovo tipo d'esteta (Il mio film d'eccezione)", non datato, ma steso di sicuro dopo l'avvento del sonoro ${ }^{7}$.

3. Pavese in una lettera alla Dowling (19/3/1950) (MASOERO, 2003, p. 23). Il 14 gennaio 1944 lo scrittore annota nel suo diario: "Noi tendiamo al dialogo, alla conversazione. Amiamo evitare le lunghe note informative (la narrazione), anzi anche queste le trasformiamo in discorso facendole in prima persona e colorite secondo il personaggio che le pronuncia. Cerchiamo insomma, nella narrativa, il teatro, ma non la scenica. Che venga dall'uso del cinema, che ci ha insegnato a distinguere tra visività e parola, che prima il teatro fondeva?" (PAVESE, 1974, p.257).

4. Nel frammentare la trama, Pavese si inserisce nella tradizione dei prosatori narranti della letteratura italiana, come registra nel suo diario, il 2 novembre 1943: "La poiesis italiana ama le grandi strutture fatte di piccoli capitoletti, di parti brevi e sugosissime - i frutti dell'albero. (Dante, i brevi canti; Boccaccio, le novelle; Machiavelli, i capitoletti delle opere maggiori; Vico, gli aforismi della Scienza Nuova; Leopardi, i pensieri dello Zibaldone, ecc. Per non parlare del sonetto)” (PAVESE, 1974, p.252). 
Nel primo dei due saggi - come osserva Mila (1958, p. 14), nel presentarli -, lo scrittore è "diligentemente impegnato a formulare una definizione estetica del fatto cinematografico". Infatti Pavese (1958b, p. 15) si scaglia contro coloro che non riescono ad andare oltre a una critica empirica di un film, a capire di trovarsi di fronte ad una nuova espressione artistica e, difettando i mezzi adeguati per giudicarla, la riducono spesso a una semplice traduzione di altre arti:

È nello schema di giudizio applicato, con più o meno sensibilità e chiarezza, da tutti i critici improvvisati in quest'arte che non ha finora teoria critica, che si può vedere quanto sia confusa e oscura la coscienza della differenza specifica dei mezzi d'espressione del cinematografo (che è poi differenza della natura delle sue creazioni) da quelli del teatro, della pittura, del romanzo, di tutte le forme d'arte insomma che appunto prestano la loro terminologia tecnica ai pasticciosi giudizi di detti recensori. Questo schema di giudizio che invariabilmente si ritrova sotto tutti i saggi di critica cinematografica è tale che sminuzza ciascun'opera in tanti diversi presunti suoi fattori (la trama, la recitazione, la messa in scena, la luce, il taglio, la fotografia, per citare i più comuni) e questi fattori considera a sé, fuori della sintesi che li dovrebbe aver annullati nell'opera [...].

In questo modo, secondo Pavese (1958b, p. 16), è dubbio

se il cinematografo inteso storicamente come congenie di mezzi tecnici atti a rappresentare è tale da permettere a questi mezzi bruti una sintesi, un'espressione, un così detto insomma fantasma poetico, che sia se stesso, soltanto nella nuova natura cinematografica e non invece una possibile meccanica traduzione di altra precedente sintesi trasposta in questa forma a scopo divulgativo.

Se insomma il cinematografo sia illustrazione o arte.

Pur risconoscendo che il cinema è ancora in gran parte illustrativo, "che esso cioè dice le cose traducendole o come se le traducesse da altre arti", Pavese non esclude che molti film portino "esempi di una nuova sensibilità cinematografica che, se non è giunta finora a creare tutta un'opera schietta, lo deve alla tradizione inceppante dei

\footnotetext{
5. In una lettera alla Dowling (8 maggio 1950), scrive Pavese: "Questa volta non includo le osservazioni. Credo che la storia (chiamiamola Gli innocenti o Le belle famiglie italiane o A questo mondo c'è giustizia) parli da sé” (MASOERO, 2003, p. 25).

6. Pavese in una lettera alla Dowling (13/5/1950) (MASOERO, 2003, p. 24).

7. Dice Pavese (1958a, p. 21) contro gli esteti: "recentemente, 'il parlato non va"'. Anche se molte pellicole sonorizzate circolavano sugli schermi della capitale già nel 1928 (dunque un anno dopo l'avvento del sonoro negli Stati Uniti), il primo film parlato italiano - La canzone dell'amore di Gennaro Righelli - è del 1930 (MARTINELLI, 1996, p.223; SAVIO, 1975, p. 56-57). Mariarosa Masoero (2003, p. 21) e Gian Piero Brunetta (1982, p. 231) segnalano altri due articoli dedicati dallo scrittore al grande schermo, rispettivamente "Per la famosa rinascita" (1927) e "Ieri e oggi", stampato sull'Unità nell'agosto 1947.
} 
falsi clichés già formatasi e all'influsso appunto della falsa concezione critica corrente". Nell'individuare la natura ancora ibrida della nuova arte, Pavese (1958b, p. 16-17, 19) biasima che fino allora "molto romanzo e più teatro cercano di farsi valere sotto il nome di cinematografo".

Nel secondo testo, Pavese (1958a, p. 20-21) disdegna coloro che, avendo scoperto in ritardo l'importanza del cinema, in nome delle sue doti artistiche, sottovalutano lo spettacolo di intrattenimento e vanno alla ricerca del "film d'eccezione":

Molta gente - la stessa che quindici, e anche dieci, anni fa non andava
al cine perché era roba da serve - adesso ha scoperto che il cinema e
un'arte e si dà quindi d'attorno - colle parole, almeno - per ridurre il
medesimo il più arte possibile, per strapparlo alle serve, alla folla, per
ammazzarlo, insomma, nei bei film d'eccezione. Ammazzarlo. Poiché,
non si ripeterà mai abbastanza che il cinematografo è un'arte da folla
e che la ragione della sua vitalità è appunto questa: che esso ha creato
un'arte nient'affatto d'eccezione, fine-ottocento o principio-novecento,
ma interamente popolare, che parla cioè a tutti i pubblici. E si capisce,
così, come i suoi primi frutti di qualche valore siano venuti dal Norda-
merica, il paese che, per la sua giovinezza e per la sua formazione unica
al mondo, ha meno divario di bisogni spirituali tra le classi e rinnova
quindi per noi, in parte, lo spettacolo di una civiltà primitiva attraverso
forme raffinate. [...]

Ora, scusino gli esteti se entro nell'autobiografia. Ma voglio dire come io, che per motivi strettamente personali, ho cominciato a frequentare cinemini da due lire e anche meno, mi sono accorto, a un certo punto, che gli schermi un tantino maculati dei locali in questione sono gli altari dove si celebrano feste d'arte, inaudite in luoghi meno popolari. Una quantità di filmetti giudicati di scarto perché non forniti di un nome famoso d'attore non rappresentanti "eccezioni" artistiche, passa in quelle traballanti macchine di proiezione. E ci sono capolavori dei più schietti.

Belle storie, in genere della casa Fox - la più odiata dagli esteti (quelli che sanno che esiste) e si capisce: è la casa di Giorgio O'Brien e di Olive Borden, - con quel tanto d'esotico che basta, qualche irresistibile macchietta, primi attori svelti, nessuna "sovrapposizione" lirica, tragedia anche, se occorre, ma ben dosata, e persino profondità psicologiche, per chi vuole pensarci: la miglior scuola americana, lo stesso che,

8 "-Non è che gli artisti siano stupidi, ma siete voi deficienti. Critici rompicoglioni che vivete solo di libri e, se andate al cinematografo, cercate la cerebralità e, se andate in giro, cercate l'interessante e non vivete la vita come gli operai o i bambini: la guardate da lontano." - fa esclamare Pavese (2002, p. 302-303) a uno dei personaggi in un racconto del 1929, "Arcadia” (Testi giovanili). 
con attori più famosi, la Metro-Goldwyn. La folla è uscita di lì. E La folla ha, per un attimo, tappato la bocca anche agli esteti.

Queste idee dell'autore piemontese sul cinema non sono presenti solo nei testi teorici, poiché le aveva espresse anche in vari passi di racconti pubblicati postumi, come in "Arcadia" (1929), in cui al "cinema d'arte" contrappone quello che, secondo lui, rivelava la vita:

Soffriva che quella ragazza invece di amare i film che a lui parevano esprimere la sua vita - quelle storie di commesse, di impiegate nelle case nitide d'America, in mezzo a una città enorme e pure fresca come una campagna - cercasse invece, e ne parlasse, le pellicole storiche coi bei vestiti, le belle pettinature, i duelli generosi e tutta la patina da cartolina illustrata. (PAVESE, 2002, p. 299) 9

Critico di una certa produzione europea, specie quella italiana ${ }^{10}$, per Pavese il cinematografo, quale arte nuova, appartiene ad un paese nuovo, l'America, come viene detto ne "L'avventuriero fallito", uno degli scritti che compongono la Trilogia delle macchine (1928):

Era partito pieno di febbre e di risolutezza salda, a vent'anni, verso l'America nuova, fanatico di quell'umanità, con una speranza ardente di vivere quella vita, assorbirla e esprimerla nell' arte nuova che doveva vestire la nuova bellezza del mondo.

E là, s'era aggirato, aveva fatto di tutto, intorno ai focolari della cinematografia, tutte le parti più umili, dall'operaio alla comparsa, aveva sofferto, faticato in silenzio, con poco cibo e meno riposo, senza tregua per anni, sperando credendo sempre. [...]

E ora, la sopravvivenza irritante degli insopportabili drammi, a lui, che nel suo lungo attrito colle folle più diverse, aveva sentito confusamente, ma recisamente, il bisogno della forma nuova, la sua cinematografia [...]. (PAVESE, 2002, p. 257, 260).

\footnotetext{
9. Vedasi anche il seguente brano di un altro racconto, "Una domenica" (PAVESE, 2002, p. 211): "In un piccolo cinematografo pieno di fumo, tra il vocio, il tanfo e gli scracchi, vidi un giovane appoggiato alla parete, immobile. Sullo schermo sporco s'illuminava in quell'istante un viso pallido, delicato, uno di quei grandi visi di giovinetta ignara, dall'aria ancor bambina, che non mancano mai in quei romanzi e a me danno insopportabilmente sui nervi perché son tanto lontani dalla realtà che mi par di conoscere".

10. Vedasi questo passo di "Vita notturna": "Passava il film Luce. - Che film seccanti fanno in Italia! - disse forte, come introduzione, Guidi e nessuno dei pochi spettatori protestò. [...] Fuori, le fotografie esposte promettevano un'avventura variopinta, mezzo esotica e mezzo di guerra.
} 
L'ammirazione giovanile per la cultura e il comportamento nordamericani ${ }^{11}$, che si proietta idealmente sulla visione che ha della sua città, Torino - "dove sono nato spiritualmente, arrivando di fuori” (PAVESE, 1974, p. 20) - si fa ancora sentire, al suo ritorno dal confino, nelle pagine de Il mestiere di vivere (25 dicembre 1937):

Ricordi come i tuoi sogni di case operaie e limpide, i tuoi corsi alberati su un prato, la tua città fredda sotto le montagne, le insegne al neon rosso di fronte alla piazza delle montagne, le domeniche erranti verso questa piazza, sui selciati, e poi il tuo lacerante sogno di compagnie piemontese-internazionali, di ragazze che vivono sole e lavorano, di plebea eleganza e serenità, e poi tutte le tue poesie del primo anno: si sono annichilati per sempre col 9 aprile? Non c'è tutta la tua giovinezza nel cinema e nella piazza Statuto? (PAVESE, 1974, p. 69).

E, di nuovo in "Arcadia", ecco l'esaltazione di un modo di vita e della sua rappresentazione non più schiavi di una mentalità ottocentesca e di moduli melodrammatici:

\begin{abstract}
I famosi grattacieli Paolo li aveva scoperti al cinematografo.
Paolo era sempre andato al cinematografo.
\end{abstract}

Da bambino, ricordava confusamente, la vita gli era stata funestata da visioni di pellicole orripilanti italiane o francesi che non lo lasciavano più dormire alla notte. Le prime attrici le ricordava tutte scarmigliate $\mathrm{e}$ tragiche, e gli attori, tutti uomini in nero - avvocati o truffatori o primi amorosi che fossero -; le scene eran cose spaventose - rapimenti, delitti, testamenti, colloqui notturni, addii di amanti nei parchi di castelli - e il tutto sempre in catapecchie cadenti o in palazzoni d'ambasciata.

E persino le storielle comiche eran così truci e grigie che Paolo preferiva non pensarci. Lasciando stare poi che tutto questo succedeva nel salone buio che ai bambini di allora faceva ancora paura. [...]

Tutto questo era molto lontano. Paolo ora aveva scoperto i grattacieli.

Era stata una sera che lui, ancora studente, strascinava in un piccolo cine un pomeriggio tedioso e freddo d'inverno. Una scena improvvisa: sul telone nebbioso, il paesaggio irreale dei colossi accatastati, geometrici e remoti.

- Chissà che boiata!

- Mah! La Fox è specialista.

- 'Sti americani pare l'abbiano fatta loro la guerra.

Questo a Guidi dispiacque. Aveva notato che tutti quelli che non sanno cosa dir d'altro han l'abitudine di prendersela coi films di guerra americani.

[- E il bello è che il primo film di guerra l'han fatto in Italia con prima attrice Diomira Jacobini che veniva sforzata da un vigliacco ufficiale austriaco, debitamente ucciso poi in un corpo a corpo dall'innamorato della seviziata.]

La sera passò lenta. I due, quasi in silenzio, seguivano, rassegnati, sul telone le vicende della solita cameriera al fronte. Ma Bruno soffiava ogni tanto, e protestò anche, con una gran voce insonnolita, quando la ragazza finì in trincea a cercare il suo amore. C'era poi 
Quel che piacque dapprima a Paolo fu l'atmosfera della scena, quella lontananza, quei lumi radi. Somigliava a ciò che da un pezzo lui andava per la città a cercare fin nelle barriere: le vedute lontane, d'inverno, i corsi interminabili dai lampioni annebbiati, e gli urti e i contatti di quella folla di operai, alla sera, affrettantesi intorno a lui trasognato. Poi, vagabondando - nelle mattinate frizzanti, nelle giornate grigie - aveva scoperto un'altra bellezza di queste barriere: le fabbriche, tutte invetriate di finestre, ritte solitarie tra case nuove, bianchissime, disseminate nei prati. [...]

Queste furono i suoi grattacieli.

[...] E allora entrava in qualche piccolo cinema e, abbandonandosi sul sedile, lasciava fissarsi gli occhi senza più pensare ed era quasi felice.

Le pellicole d'incubo erano finite da tempo. Ora dominavano i film d'America. Giungevano nei cinematografi di barriera dopo lunghe proiezioni nei saloni di lusso. Ma Paolo preferiva vederle ora e non solo per economia. Gli piaceva mescolarsi a quel pubblico - ragazze senza cappello, operai - e di là levare il capo e sognare.

Vivevano sul telone uomini gagliardi dal largo riso splendente e donne giovani, incredibilmente giovani. In avventure mortali o in casi dell'esistenza d'ogni giorno questi protagonisti erano sempre uguali a se stessi. Paolo si sentiva rivivere in una vita più sana e più serena, una vita semplice e inimitabile, la vera vita dei paesaggi che lui andava scoprendo agli orli della città. E ognuna di quelle storie era una sua avventura che in quel silenzio magico, il silenzio del pensiero e della fantasia, viveva in un buio, come nella sua anima stessa. (PAVESE, 2002, p. 289-291).

Le piccole sale cinematografiche, soprattutto i locali di barriera - dove davano i "film d'America", con la loro "trama semplice e vistosa", come si legge ne "La trilogia delle macchine: III - Il pilota malato" (PAVESE, 2002, p. 276) -, site in quelle strade che sconfinavano nella campagna, sono le predilette dello scrittore. In esse coniuga la scoperta di una nuova società e quella della realtà popolare della sua città, come in questo brano di “Arcadia” (Ciau Masino) (1929-1932):

Masino si salvava al cinema. Questo è stato per la nostra giovinezza un gran manna. Le settimane interminabili venivan così rotte in tante ore

un comico fesso che adoperava una pipa per rivoltella. Guidi secondava il malumore di Bruno e fu lui che a un certo punto propose di andarsene senza aspettare il resto. [...]

Giravano un angolo, quando Bruno, tutto da sé, scoppiò a ridere.

- Cos'hai?

- Penso a quel film - quando cerca la pipa. Ma lo sai che è ben fesso? Come fanno a lavorare sul serio là sopra?

- Va' là che anche in Italia sono in gamba, - disse Guidi. - Tra Maciste e Napoli!” (PAVESE, 2002, p. 311-312, 997-998). Il brano fra parentesi quadre è stato soppresso dalla pubblicazione. In esso lo scrittore si riferisce a La leggenda del Piave (1924) di Mario Negri, che commuove gli spettatori ma non i critici. Negli anni Venti, il regime fascista si avvale solo dei documentari de L'Unione per la Cinematografia Educativa (LUCE) quale strumento di propaganda, anche se produce alcuni film a sfondo patriottico. Le pellicole di 
trascorse in un mondo ormai famigliare, eppure sempre affascinante.

A Masino piacevano i filmetti d'America. C'era anche, innanzi a questi, l'orgoglio di scoprir sempre qualcosa, di vivere in un mondo nuovo. Questi film sono fatti apposta per i locali di barriera. Masino usciva al pomeriggio, camminava per vie interminabili, oltre i corsi, oltre le grandi vie del centro, fino a Dora, fino alle regioni dei prati, tra le case operaie, dove tutto è recente e in costruzione - grandi case nel cielo coi fianchi lisci, tagliati, pronti a riceverne altre, all'infinito - respirava quell'aria più aperta, più frizzante, guardava i negozietti lucidi, provinciali e immaginava di vivere quella vita, di soffrire quel lavoro - le fabbriche, le acque luride, le erbe bruciacchiate, l'orizzonte.

I film americani. Costava poco entrare in quei cinemi e si vedevano le cose più belle. Buck Jones, Giorgio O'Brien, Olive Bordeu, Sue Carol - il mare, il Pacifico, le foreste, le navi. Ma sopratutto le cittadine dell'America, quelle case nitide in mezzo alle campagne, quella vita schietta e elementare. Tutto era bello. Gli uomini, individui sicuri, forti, con un sorriso tra i denti, pugni sodi ed occhio aperto. Le ragazze, sempre le stesse dai villaggi alle metropoli, corpo chiaro, volto allegro, sereno, anche in mezzo alle sventure. Si usciva leggeri da quei film. $\mathrm{Nel}$ centro dicevano che eran cose banali senz'effetto e senza vita, ma a Masino pareva proprio d'imparare a vivere assistendo a quelle scene.

Il pubblico anche, piaceva a Masino. Se si passava sopra alla puzza e alle pulci - pregiudizi - quelli avevan tutta l'aria di gente più seria e degna del mondo. (PAVESE, 2002, p. 371-372) ${ }^{12}$.

Un po' più giovane di Pavese, Mario Monicelli ha ancora ben presente nella memoria quelle sale popolari che frequentava da bambino, come dichiara a Nello Ajello (2009, p. 112):

dal 1920 in poi, mia madre [...], quasi ogni pomeriggio prendeva me e mio fratello Franco, d'un paio d'anni più grande di me, e ci portava ai cinema. Passavamo mezza giornata in quelle sale fumose, sporche, affollatissime, con gente seduta per terra. Donne che allattavano i bambini fissavano le scene che passavano sul lenzuolo. Era come se in sala si esibisse una seconda compagnia d'arte. Si faceva il tifo per i "nostri" che stavano arrivando, si difendeva la ragazza innocente e si condannava quella "perversa", s'inveiva contro il traditore e il nemico. Ci si

\footnotetext{
Maciste, in gran parte dirette da Guido Brignone, entusiasmano il grosso pubblico. Le sceneggiate cinematografiche, in specie quelle di Ubaldo Maria Del Colle e Elvira Notari, ispirate dalle canzonette napoletane, sebbene snobbate dalla critica e nel resto dell'Italia, hanno un enorme successo nel Meridione, nelle isole e presso le comunità italiane delle Americhe (MARTINELLI, 1996, p. 39-40, 7-8, 215). 11. Non va dimenticato che Pavese è tra gli intellettuali che negli anni Trenta e Quaranta divulgano in Italia opere di autori come Erskine Caldwell, John dos Passos, William Faulkner Ernst Hemingway, Sinclair Lewis, Edgar Lee Masters, Herman Melville, Gertrude Stein, John Steinbeck, e che alla cultura statunitense dedica anche una serie di riflessioni scritte fra il 1930 e il 1950 e riunite ne La letteratura americana e altri saggi. Da ricordare anche i testi di Mario Soldati (America primo amore, 1935), Emilio Cecchi (America amara, 1938), Giaime Pintor (Il sangue d'Europa. Scritti politici e letterari, 1939-1943) e l'antologia Americana (1942) a cura di Elio Vittorini.
} 
commuoveva. Si gioiva. Si urlava e piangeva con molto rumore. Posso dire di non aver mai visto film più sonori dei film muti. Ne ero conquistato.

In quegli anni, per molti giovani, cinematografo voleva dire cinema americano, con quei "filmetti ottimistici dell'American way of life [...] e del keep smiling", come ricorda Mila (1958, p. 14):

Sarà stata $[\ldots]$ la retorica dell' antiretorica; certo è che questa infatuazione per il cinema americano, inteso in maniera giovanile come una miniera di modelli di comportamento e come un ideale di vita, faceva parte d'un nostro costume e d'una nostra tenace volontà di antiletteratura: in una parola, faceva parte del nostro antidannunzianesimo ${ }^{13}$.

L'interesse di Pavese verso il cinema americano come un fatto di costume ci porta a pensare quanto ne siano debitrici alcune produzioni italiane che hanno preparato l'avvento del neorealismo cinematografico, da Gli uomini, che mascalzoni! (1932) di Mario Camerini a Quattro passi fra le nuvole (1942) di Alessandro Blasetti: esso ha insegnato a camminare, a parlare, a comportarsi in una società contemporanea. Ossia i film d'America hanno aiutato a svecchiare una cultura e a superare un cinema ancor troppo teatrale o letterario.

\section{Referências bibliográficas}

AJELLO, N. Mondo Monicelli. In L'Espresso, Roma, LV (19): 112-114, 14 mag. 2009.

FOFI, G. Come in uno specchio. Roma: Donzelli, 1995.

MARTINELLI, V. Il cinema muto italiano: i film degli anni venti (1924-1931). Roma/Torino: Centro Sperimentale di Cinematografia/Nuova ERI-Edizioni RAI, 1996.

MASOERO, M. Cronologia. In PAVESE, C. Tutti i racconti. Torino: Einaudi, 2002, p. XCICIII.

Postfazione. In PAVESE, C. Il diavolo sulle colline: soggetto cinematografico. Pistoia: Via del Vento Edizioni, 2003, p. 21-29.

12. Le sale di seconda visione e quelle di periferia - quei "cinema da una lira", come scrive in "Ospedale" - sono evocati inoltre in "Vita notturna" e ne "La trilogia delle macchine: I - L'avventuriero fallito" (PAVESE, 2002, p. 391, 311, 263): "Entrava a volte, tediato in uno dei piccoli cinemi di barriera, dove c'è fumo, puzzo e sputi e i ragazzi gridano, appassionandosi all'azione".

13. Comunque, come segnala Goffredo Fofi (1995, p. 77), la coscienza dello sgretolarsi del mito americano era già riscontrabile nella cinematografia locale, specialmente ne La folla (The crowd, 1928), “impressionante illustrazione dell'alienazione dell'uomo-massa". Il film di King Vidor è stato uno dei più amati da Pavese, come si deduce dai saggi critici e da un altro brano di "Vita notturna": "[Guidi] Pensò che in fondo tutta la sua vita intellettuale era ridotta al cinematografo. E non osava difendersi. 
MILA, M. Due inediti di Pavese. In Cinema Nuovo, Roma,VII (134): 14, lug.-ago. 1958.

PAUTASSO, S. Guida a Pavese. Milano: Rizzoli, 1980.

PAVESE, C. Di un nuovo tipo d'esteta (Il mio film d'eccezione) In Cinema Nuovo, Roma, VII (134): 20-21, lug.-ago. 1958a.

. Due soggetti cinematografici inediti di Cesare Pavese: Il diavolo sulle colline,

Breve libertà. In Cinema Nuovo, Roma, VIII (141): 389-400, set.-ott. 1959.

. La letteratura americana e altri saggi. Torino: Einaudi, 1978.

. Il mestiere di vivere (Diario 1935-1950). Torino: Einaudi, 1974.

. I problemi critici del cinematografo. In Cinema Nuovo, Roma, VII (134): 15-19,

lug.-ago. 1958 b.

. Tutti i racconti. Torino: Einaudi, 2002.

SAVIO, F. Ma l'amore no: realismo, formalismo, propaganda e telefoni bianchi nel cinema italiano di regime (1930-1943). Milano: Sonzogno, 1975.

- Ma la Folla non era male, - disse -. Dentro di sé lo credeva un capolavoro. [...]

Guidi pensò ora che forse tutta la sua supinità nella vita gli veniva dal troppo cinematografo. Tutte le avventure, le donne più belle, che cosa importava più la realtà? [...]

Ma no, già da bambino, ricordava, era stato inetto e rassegnato. La Folla. Quella la sua incapacità a vivere. E la vita toglie di mezzo. Uno dei tanti: perduto come uno dei tanti.

La Folla. Val la pena di urlare?

Un uomo la vince. Charles Murray, un giovane dagli occhi irrequieti. La leggenda della Folla. Lui che, in persona, mendica per anni, inutilmente, un posto di comparsa e poi di colpo piace a King Vidor e diventa il protagonista del gran film, uno dei tanti" (PAVESE, 2002, p. 313-314). 